\title{
Duale Führungssysteme empfehlenswert
}

\author{
Stephanie Krekeler
}

Ein bedeutsames Element von Corporate Governance stellt die Trennung von Geschäftsführung und Aufsicht dar. Duale Führungsstrukturen brauchen jedoch eine solide strukturelle Grundlage - und bei allen Verantwortlichen den Willen zur vertrauensvollen Zusammenarbeit im Führungsalltag.

Neue Qualitätsanforderungen in Gesetzen, immer knapper werdende öffentliche und kirchliche Mittel sowie zunehmender Wettbewerb, Marktorientierung und verstärkte Konkurrenz - vor allem mit gewerblichen Anbietern - stellen immer höhere Ansprüche an die Wettbewerbsfähigkeit und Wirtschaftlichkeit sozialer Träger. Die gestiegenen Anforderungen fordern immer bessere Träger-, Organisations-, Führungs- und Aufsichtsstrukturen sozialwirtschaftlicher Unternehmen, damit diese sich am Markt behaupten können. Vor diesem Hintergrund ist in den letzten Jahren zunehmend die Einrichtung von Kontrollund Aufsichtsgremien in den Fokus gerückt.

Sinnvoll erscheint die Einrichtung dualer Führungsstrukturen insbesondere vor dem Hintergrund, dass die Gesellschafter einer GmbH oder die Mitglieder eines Vereines (die Begrifflichkeit steht hierbei in Abhängigkeit zu der jeweiligen Rechtsform des Unternehmens) oftmals nicht über den erforderlichen zeitlichen Rahmen verfügen, um selbst eine laufende Aufsichtsfunktion gegenüber der Geschäftsführung des Unternehmens wahrzunehmen. Darüber hinaus verfügen die Verantwortlichen oft nicht immer über die notwendige Fachkompetenz sowie oftmals nicht über alle erforderlichen Informationen, um eine laufende Aufsichtsfunktion gegenüber der Geschäftsführung zu gewährleisten. Da jedoch die gesteigerten Forderungen an die Unternehmensführung eine Optimierung entsprechender Unternehmensstrukturen inzwischen unumgänglich machen, bietet sich die Errichtung eines separaten Aufsichtsgremiums an, das diese Aufgaben bestmöglich wahrnehmen kann.

\section{Das duale Führungssystem im Lichte verschiedener Rechtsformen}

Die einschlägigen gesetzlichen Vorschriften erfordern die Einrichtung eines Aufsichtsgremiums rechtlich zwingend nur bei einigen wenigen Rechtsformen; bei vielen Unternehmensformen ist ein Aufsichtsrat hingegen lediglich als fakultatives Gremium vorgesehen. Gesetzlich vorgeschrieben ist ein Aufsichtsrat beispielsweise für Aktiengesellschaften und Genossenschaften. Da diese

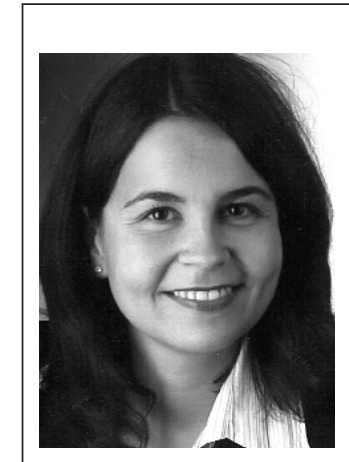

Stephanie Krekeler ist zugelassene Rechtsanwältin bei der Rechtsanwaltskammer Köln. Nach ihrer beruflichen Tätigkeit in der Rechtsabteilung eines in Köln ansässigen Wirtschaftsunternehmens ist sie nunmehr im Beratungsbereich der Solidaris Revisions- $\mathrm{GmbH}$ Wirtschaftsprüfungsgesellschaft Steuerberatungsgesellschaft und für die Sozietät Fuchs Münzel Scherff in Köln tätig. Einen Schwerpunkt ihrer Tätigkeit bildet dabei die anwaltliche Beratung von Unternehmen im Gesundheitsund Sozialwesen insbesondere in Fragestellungen des Gesellschafts-, Arbeits- und Krankenhausrechts. Internet http://www.solidaris.de

Gesellschaftsformen bei sozialtätigen Unternehmen jedoch in der Praxis eine untergeordnete Rolle spielen, sind sozialtätige Unternehmen im Ergebnis rechtlich nur selten zur Errichtung eines Aufsichtsgremiums verpflichtet. Bei den im Gesundheits- und Sozialwesen häufiger anzutreffenden Rechtsformen der $\mathrm{GmbH}$, des Vereines, der Stiftung oder der Körperschaft des öffentlichen Rechts handelt es sich um Gesellschaftsformen, denen gemein ist, dass ein Aufsichtsrat nicht zwingend vorgesehen ist.

Gleichwohl empfiehlt sich die Errichtung eines Aufsichtsgremiums aus den eingangs genannten Gründen auch in Unternehmen, in denen aufgrund ihrer Rechtsform ein Aufsichtsgremium nicht verpflichtend vorgesehen ist. Insbesondere in Unternehmen, die in größerem Ausmaße oder zunehmend wirtschaftlich tätig sind, erscheint ein Aufsichtsgremium zur verbesserten Unternehmensführung sachgerecht. Der Grundsatzentscheidung, ob sein Aufsichtsgremium neben dem geschäftsführenden und vertretungsberechtigten Organ errichtet werden soll, sollte bei der Ausgestaltung des Unternehmens und dessen Organen besonderes Augenmerk gewidmet werden.

\section{Besetzung, Aufgaben und Ziele eines Aufsichtsgremiums}

Soll ein (fakultativer) Aufsichtsrat eingerichtet werden, empfiehlt es sich, die Bildung, Besetzung und Aufgaben 
des Aufsichtsrates in der Satzung oder einer zusätzlichen Geschäftsordnung festzuschreiben und diese je nach Vorstellung über die Ausgestaltung des Gremiums mehr oder minder detailliert $\mathrm{zu}$ regeln.

Die Konzeption des fakultativen Gremiums ist frei gestaltbar: Neben der Aufgabenzuordnung ist die Zahl der Personen, aus denen sich der Aufsichtsrat zusammensetzen soll, frei bestimmbar. Regelungen hierzu sollten ebenfalls im Gesellschaftsvertrag oder einer separaten Geschäftsordnung für den Aufsichtsrat beschrieben und getroffen werden. Grundsätzlich variiert die Anzahl der Aufsichtsratsmitglieder von Unternehmen zu Unternehmen und ist insbesondere von der Größe und Ausrichtung der Gesellschaft abhängig. In der Praxis hat sich jedoch eine Stärke des Aufsichtsgremiums von fünf bis sieben Personen etabliert und als sinnvoll erwiesen.

Die Auswahl und Benennung der Aufsichtsratsmitglieder sollte inhaltlich maßgeblich davon mitbestimmt sein, dass die Aufsichtsratsmitglieder über die zur ordnungsgemäßen Wahrnehmung ihrer Aufgaben erforderlichen Fähigkeiten, Fachkenntnisse oder Sachkunde verfügen sollten. Darüber hinaus sollten sie ausreichend unabhängig sein, um ihre Kontroll- oder Beratungsaufgaben sachgerecht und kompetent wahrnehmen zu können.

\section{Umsetzung dualer Führungsstrukturen in der täglichen Praxis}

Soweit ein Aufsichtsgremium eingerichtet wurde, erscheint es sinnvoll, dessen Arbeit sowie die Integration des Gremiums in das Unternehmen bereits in der täglichen Praxis zu fördern und zu unterstützen. Dabei kann bereits die Umsetzung einiger weniger praktischer Maßnahmen dazu beitragen, die eingerichteten dualen Führungsstrukturen schnellstmöglich zu etablieren und ihre Funktionsfähigkeit zu verbessern:

- Zusammenwirken von Geschäftsführung und Aufsichtsgremium: Im Rahmen des dualen Führungsmodells ist ein enges und vertrauensvolles Zusammenwirken von Geschäftsführung und Aufsichtsgremium zum Wohle des Unternehmens unerlässlich. Dies bezieht sich insbesondere auf die strategische Ausrichtung der Einrichtung, die in einem regelmäßigen Turnus Gegenstand einer konstruktiven Diskussion zwischen den Beteiligten sein sollte.

- Information des Aufsichtsgremiums: Von besonderer Bedeutung für die Zusammenarbeit ist zudem die regelmäßige und umfassende Unterrichtung des Aufsichtsgremiums durch die Geschäftsführung. Die Erfüllung dieser Informations- und Berichtspflichten obliegt zwar grundsätzlich der Geschäftsführung, es empfiehlt sich aber, den notwendigen Umfang vom Aufsichtsgremium detailliert definieren zu lassen. Das Aufsichtsorgan sollte daher in einer Informationsordnung festlegen, welche Informationen es in welcher Qualität, Quantität und in welchen zeitlichen Abständen benötigt und welche Vorbereitungszeit vor einer Aufsichtsratssitzung einzuplanen ist. Da nicht immer alle Informationen schriftlich aufbereitet und rechtzeitig übermittelt werden können, ist ferner gegebenenfalls die zusätzliche mündliche Berichterstattung der Geschäftsführung not- wendig, um Hinweise auf einen möglichen Handlungsbedarf zu erhalten. Zusätzlich zu diesen Mindestvorgaben ist anzuraten, dem Aufsichtsgremium weitere Informationen in Form permanenter, periodischer oder fallspezifischer Berichte zuzuleiten, beispielsweise die turnusmäßige Berichterstattung über nicht finanzielle Indikatoren sowie Sonderberichte zur Markt- und Wettbewerbssituation, zu zustimmungspflichtigen Geschäften, zur Risikosituation, zum Risikomanagement sowie Berichte der internen Revision und externer Aufsichtsund Prüfungsbehörden.

- Zustimmungspflichtige Rechtsgeschäfte: Von großer Bedeutung in der Zusammenarbeit beider Organe ist die Bezeichnung der Geschäfte, die nur mit Zustimmung des Aufsichtsgremiums vorgenommen werden dürfen (zustimmungspflichtige Geschäfte). Diese müssen sich im Rahmen des Gesellschaftsvertrages oder der Satzung der Einrichtung halten und sollten darüber hinaus in einer Geschäftsordnung für die Geschäftsführung detailliert niedergelegt werden. Dabei sollte der Katalog zustimmungspflichtiger Geschäfte, der alle für die Existenz und Fortführung der sozialtätigen Unternehmen wichtigen oder besonders risikobehafteten Geschäfte zu beinhalten hat, jeweils an wesentlich veränderte Situationen oder wirtschaftliche Veränderungen angepasst werden.

\section{Resümee}

Die Errichtung eines Aufsichtsgremiums ist in den üblicherweise anzutreffenden Gesellschaftsformen sozialtätiger Unternehmen nur in den seltensten Fällen gesetzlich vorgeschrieben.

Unabhängig hiervon erscheint die Installation eines entsprechenden Aufsichtsgremiums gleichwohl zumeist zweckmäßig, um Kontroll- und Leitungsstrukturen der Unternehmen zu installieren oder zu optimieren. Insbesondere im Hinblick auf die gestiegenen wirtschaftlichen und gesetzlichen Anforderungen auch an sozialtätige Unternehmen sowie deren zunehmende wirtschaftliche Betätigung erscheint die Einrichtung effizienter Aufsichtsgremien fast unerlässlich. Werden Aufsichtsorgane errichtet, so ist es Aufgabe der Führungskräfte und der Geschäftsführung, die Integration des Aufsichtsgremiums in die Einrichtung zu fördern und eine vertrauensvolle $\mathrm{Zu}$ sammenarbeit mit diesem vorzuleben.

Allgemein kann die Effizienz des Aufsichtsgremiums bereits durch ein Bewusstsein für den Hintergrund der Einrichtung desselben sowie durch einige flankierende Maßnahmen zusätzlich unterstützt werden, beispielsweise durch die Zusammenarbeit mit dem Abschlussprüfer, die Unterstützung durch ein funktionierendes internes Überwachungssystem, die qualifizierte Besetzung des Organs. Auf diese Weise lässt sich bereits durch einige wenige Maßnahmen eine fruchtbringende Ausgestaltung und Stärkung der unternehmensinternen Leitungs- und Kontrollstrukturen erreichen. 